\title{
Genetic Divergence Analysis in Advanced Breeding Lines of Groundnut (Arachis hypogaea L.)
}

\author{
E. Aruna Kumari*, K. John, D. Mohan Reddy and P. Latha
}

S.V. Agricultural College, Tirupathi- 517502, Andhra Pradesh, India

*Corresponding author

\section{A B S T R A C T}

\section{Keywords}

Groundnut, Cluster, D2 and genetic divergence

Article Info

\section{Accepted:}

12 April 2021

Available Online:

10 May 2021
Genetic diversity among 30 advanced breeding lines of groundnut were estimated using Mahalanobis D2 statistic for 21 characters. The analysis of variance revealed significant differences among the advanced breeding lines for all characters. Based on Tocher's method, 30 advanced breeding lines were grouped into ten clusters. Cluster II is the largest cluster with seven genotypes. Cluster III and IV contain six genotypes and cluster I contain four genotypes, cluster IX contain two genotypes remaining clusters (V, VI, VII, VIII and X) are monogenotypic clusters, this grouping indicated considerable diversity among the breeding lines. Intra-cluster distance is maximum in cluster IV (138.29) followed by cluster II (105.35), cluster IX (86.77), cluster III (85.87) and cluster I (60.44). Highest inter-cluster distance was observed between cluster VIII and IX (491.25) followed by cluster IX and X (472.37) and cluster III and IX (457.84) indicating the existence of high diversity within and between clusters. Hence the genotypes from these clusters can be utilized as potential parents and crossing among them would be suggested to generate a wide range of variability for effective selection for improvement of various characters.

\section{Introduction}

Groundnut is one of the important oilseeds crop grown among countries of the world. It is native to South America and it belongs to the family 'Leguminosae'. It is a self-pollinated crop, allotetraploid with diploid chromosome number $2 \mathrm{n}=40$. It has wide variety of uses viz., kernel directly used for table purposes or can be crushed for oil, vine with leaves as fodder and shell can be made to particleboard. It contains $36-54 \%$ of edible oil, $22-36 \%$ of easily digestible protein and $18 \%$ of carbohydrates in its seeds. Groundnut oil contains 46 and 32 percent of monounsaturated fatty acids (MUFA) and polyunsaturated fatty acids (PUFA) respectively. Apart from this, groundnut kernels contain many health enhancing nutrients such as minerals, antioxidants, 
vitamins and are rich in mono-unsaturated fatty acids. They also contain antioxidants like p-coumaric acid and resveratrol, vitamin $\mathrm{E}$ and many important B-complex groups of thiamine, pantothenic acid, vitamin B-6, folates and niacin. As groundnut is an important oilseed crop used for confectionary purpose, there is a need to improve the quality traits of groundnut. Value addition through quality enhancement will earn huge foreign exchange. Hence selection for quality traits in groundnut is necessary in breeding programmes.

The D2 statistic for multivariate analysis has been successfully used to select divergent genotypes in order to exploit heterosis and for bringing together higher frequency of desirable genes in the segregates. Thus, the knowledge of genetic variability and genetic divergence in combination with character association allows a breeder to select suitable and divergent genotypes for their use in breeding strategies and to formulate a suitable selection scheme.

\section{Materials and Methods}

The present investigation was carried out during kharif, 2018 at Regional Agricultural Research Station, Tirupati. The experimental material comprised of 30 groundnut genotypes which were raised in Randomised Block Design, each entry being sown in three rows of $5 \mathrm{~m}$ length with a spacing of $30 \times 10 \mathrm{~cm}$. The observations were recorded on the basis of five randomly selected representative plants from each replication for physiological, yield and quality traits viz., days to $50 \%$ flowering, days to maturity, SPAD chlorophyll meter reading at 45 DAS, specific leaf area at 45 DAS, plant height, number of primary branches per plant, number of secondary branches per plant, number of mature pods per plant, 100-pod weight, 100-kernel weight, shelling per cent, sound mature kernel per cent, dry haulms yield per plant, harvest index, kernel yield per plant, oil content, protein content, sucrose content, total soluble sugars, total free amino acids and pod yield per plant. Days to $50 \%$ flowering and days to maturity were recorded on plot basis. The genetic diversity between genotypes was worked out using Mahalanobis $D^{2}$ (1936) extended by Rao (1952). On the basis of D2 values the genotypes were grouped into clusters according to Tocher's method (Rao, 1952). The method of Singh and Chaudhary (1977) were used to calculate the intra and intercluster distances. All the statistical calculations were done using indostat computer software.

\section{Results and Discussion}

The 30 genotypes of groundnut were grouped into ten clusters based on D2 value (Table 1). Among the clusters, cluster II is the largest cluster with seven genotypes followed by cluster III \& IV contain six genotypes and cluster I with four genotypes, cluster IX contain two genotypes whereas remaining clusters (V, VI, VII, VIII and X) were monogenotypic clusters. This grouping indicated considerable diversity among the genotypes.

The inter-cluster distance (Table 2) was larger than the intracluster distance which indicated that greater diversity is present among the genotypes of distant group \{Hampannavar and Khan (2018), Raghuwanshi et al., (2016), Zaman et al., (2010)\}. The inter-cluster distance analysis shows that the maximum divergence was observed between cluster VIII and IX (491.25) followed by cluster IX and X (472.37) and cluster III and IX (457.84). So, the genotypes in these clusters can be utilized for selection of parents for hybridization. Similar results were also suggested by Choudhary et al., (1998). The intra cluster distance was not present in cluster V, VI, VII, VIII and $\mathrm{X}$ as these clusters are mono- 
genotypic clusters. Based on inter cluster distances the clusters VIII vs IX, IX vs X, III vs IX, I vs IX, II vs VIII, VI vs VIII and VII vs VIII were found to be divergent in the decreasing order of their magnitude.

Hence, genotypes of these clusters could be utilized as parents and crossing among them would result in heterotic expression for yield components. The Cluster mean value(Table 3)for different clusters indicated that cluster I recorded the highest cluster mean for early maturity, 100-kernel weight, 100-kernel weight, shelling percent, sound mature kernels per plant, dry haulms yield per plant, oil content, sucrose content, total free amino acids and total soluble sugars. Cluster IV, VI, VIII and IX was the best source for kernel yield per plant, pod yield per plant, number of mature pods per plant, sound mature kernel percent and oil content. Cluster $\mathrm{X}$ was the best source for protein content and sucrose content. Cluster I and III was best source for days to $50 \%$ flowering and days to maturity. Contribution of character towards divergence (Table 4) was observed maximum in total free amino acids followed by number of secondary branches per plant, sound mature kernel percent, sucrose content, specific leaf area, total soluble sugars, 100-pod weight, number of mature pods per plant, pod yield, 100kernel weight, dry haulms yield per plant, kernel yield, protein content.

The present findings for the yield characters are in conformity with those reported earlier in groundnut by Vasanthi et al., (2015), Venkatesh et al., (2016), Sardar et al., (2017) and Saritha et al., (2018). Sardar et al., (2017) also recorded similar result for least performance of shelling percent to genetic divergence. However, the differences were clearer for kernel yield per plant followed by pod yield per plant, biological yield per plant, 100-kernel weight and number of pods per plant.

The present findings are in conformity with those reported earlier in groundnut (Vasanthi et al., (2015) and Saritha et al., (2018). The greatest contribution of 100-kernel weight towards divergence was also earlier reported by Sudhakar et al., (2008), Venkateswarulu et al., (2011), Venkatesh et al., (2016), Ganvit et al., (2018) and Namrata et al., (2018).It has been suggested that the character with maximum contribution towards divergence should be given importance for undergoing hybridization programme.

Considering the cluster distances and cluster means in the present investigation, emphasis should be given to genotypes belonging cluster III, VIII, IX, and $X$ can be recommended for use in the breeding programme for the development of transgressive segregants for physiological, yield and quality traits.

Based on genetic divergence studies involving 30 advanced breeding lines of groundnut, the following genotypes were suggested for successful hybridization programme. The genotypes viz., TCGS-1888 (cluster VIII), TCGS-1881 (cluster IX), TCGS-1845 (cluster IX), TCGS-2166 (cluster X) and from cluster III (TCGS-1899, TCGS-2153, TCGS-2184, TCGS-1508, TCGS-1809, TCGS-1813) can be recommended for use in the breeding programme for the development of transgressive segregants for physiological, yield and quality traits. 
Table.1 Clustering of advanced breeding lines of groundnut based on Tocher's method

\begin{tabular}{|c|c|c|}
\hline Cluster & $\begin{array}{c}\text { Number of } \\
\text { Genotypes }\end{array}$ & Genotypes \\
\hline I & 4 & TCGS-1820, TCGS-1904, TCGS-1910 and TCGS-1917. \\
\hline II & 7 & $\begin{array}{r}\text { TCGS-1818, TCGS-1823, TCGS-1824, TCGS-1825, TCGS- } \\
\text { 1895, TCGS-1915 and TCGS-2163. }\end{array}$ \\
\hline III & 6 & $\begin{array}{r}\text { TCGS-1508, TCGS-1809, TCGS-1813, TCGS-1899, TCGS- } \\
\text { 2153 and TCGS-2184. }\end{array}$ \\
\hline IV & 6 & TCGS-1829, TCGS-1839, TCGS-1901, TCGS-1903, TCGS- \\
& & 2160 and TCGS-2174. \\
\hline $\mathbf{V}$ & 1 & TCGS-2207 \\
\hline VI & 1 & TCGS-1884 \\
\hline VII & 1 & TCGS-1804 \\
VIII & 1 & TCGS-1888 \\
IX & 2 & TCGS- 1881 and TCGS-1845. \\
\hline $\mathbf{X}$ & 1 & TCGS-2166 \\
\hline
\end{tabular}

Table.2 Inter and Intra cluster (diagonal) average of $\mathrm{D}^{2}$ and $\mathrm{D}$ values (in parentheses) of advanced breeding lines of groundnut

\begin{tabular}{|c|c|c|c|c|c|c|c|c|c|c|}
\hline Cluster & I & II & III & IV & V & VI & VII & VIII & IX & $\mathbf{X}$ \\
\hline I & $\begin{array}{l}60.44 \\
(7.77) \\
\end{array}$ & $\begin{array}{c}349.24 \\
(18.68)\end{array}$ & $\begin{array}{l}192.26 \\
(13.86)\end{array}$ & $\begin{array}{l}161.62 \\
(12.71)\end{array}$ & $\begin{array}{r}199.99 \\
(14.14)\end{array}$ & $\begin{array}{l}349.11 \\
(18.68)\end{array}$ & $\begin{array}{c}326.78 \\
(18.07)\end{array}$ & $\begin{array}{l}134.61 \\
(11.60)\end{array}$ & $\begin{array}{l}429.50 \\
(20.72)\end{array}$ & $\begin{array}{r}174.99 \\
(13.22)\end{array}$ \\
\hline II & & $\begin{array}{l}105.35 \\
(10.26)\end{array}$ & $\begin{array}{l}212.66 \\
(14.58)\end{array}$ & $\begin{array}{l}288.65 \\
(16.98)\end{array}$ & $\begin{array}{l}158.64 \\
(12.59)\end{array}$ & $\begin{array}{l}124.95 \\
\text { (11.17) }\end{array}$ & $\begin{array}{l}226.38 \\
(15.04)\end{array}$ & $\begin{array}{l}428.75 \\
(20.70)\end{array}$ & $\begin{array}{l}352.69 \\
\text { (18.78) }\end{array}$ & $\begin{array}{l}179.56 \\
(13.4)\end{array}$ \\
\hline III & & & $\begin{array}{l}85.87 \\
(9.26)\end{array}$ & $\begin{array}{l}262.55 \\
(16.20)\end{array}$ & $\begin{array}{l}181.45 \\
(13.47)\end{array}$ & $\begin{array}{c}182.44 \\
(13.50)\end{array}$ & $\begin{array}{c}337.84 \\
(18.38)\end{array}$ & $\begin{array}{l}185.98 \\
(13.63)\end{array}$ & $\begin{array}{l}457.84 \\
(21.39)\end{array}$ & $\begin{array}{l}152.79 \\
(12.36)\end{array}$ \\
\hline IV & & & & $\begin{array}{l}138.29 \\
\text { (11.75) }\end{array}$ & $\begin{array}{l}202.47 \\
\text { (14.22) }\end{array}$ & $\begin{array}{l}279.15 \\
(16.70)\end{array}$ & $\begin{array}{l}242.49 \\
\text { (15.57) }\end{array}$ & $\begin{array}{l}203.27 \\
(14.26)\end{array}$ & $\begin{array}{l}273.39 \\
\text { (16.53) }\end{array}$ & $\begin{array}{r}247.11 \\
(15.71)\end{array}$ \\
\hline V & & & & & $\begin{array}{c}0.00 \\
(0.00)\end{array}$ & $\begin{array}{l}233.15 \\
(15.26)\end{array}$ & $\begin{array}{l}81.68 \\
(9.03)\end{array}$ & $\begin{array}{l}264.78 \\
(16.27)\end{array}$ & $\begin{array}{l}237.97 \\
(15.42)\end{array}$ & $\begin{array}{r}181.77 \\
(13.48)\end{array}$ \\
\hline VI & & & & & & $\begin{array}{c}0.00 \\
(0.00)\end{array}$ & $\begin{array}{c}328.82 \\
(18.13)\end{array}$ & $\begin{array}{l}426.47 \\
(20.65)\end{array}$ & $\begin{array}{l}336.82 \\
(18.35)\end{array}$ & $\begin{array}{l}177.38 \\
(13.31)\end{array}$ \\
\hline VII & & & & & & & $\begin{array}{c}0.00 \\
(0.00)\end{array}$ & $\begin{array}{l}402.22 \\
(20.05)\end{array}$ & $\begin{array}{l}133.83 \\
(11.56)\end{array}$ & $\begin{array}{c}310.92 \\
(17.63)\end{array}$ \\
\hline VIII & & & & & & & & $\begin{array}{c}0.00 \\
(0.00)\end{array}$ & $\begin{array}{l}491.25 \\
(22.16)\end{array}$ & $\begin{array}{l}342.20 \\
(18.49)\end{array}$ \\
\hline IX & & & & & & & & & $\begin{array}{l}86.77 \\
(9.31)\end{array}$ & $\begin{array}{l}472.37 \\
(21.73)\end{array}$ \\
\hline $\mathbf{X}$ & & & & & & & & & & $\begin{array}{c}0.00 \\
(0.00)\end{array}$ \\
\hline
\end{tabular}


Table.3 Cluster means for physiological, yield, its attributes and quality characters in 30 advanced breeding lines of groundnut

\begin{tabular}{|c|c|c|c|c|c|c|c|c|c|c|c|c|}
\hline $\begin{array}{c}\text { Cluster } \\
\text { No. }\end{array}$ & DF & DM & SCMR & SLA & PH & NPB & NSB & NMP & $\begin{array}{c}\mathbf{1 0 0}- \\
\text { PW }\end{array}$ & $\begin{array}{c}\text { 100- } \\
\text { KW }\end{array}$ & SP & SMK\% \\
\hline I & 30.92 & 109.50 & 43.33 & 210.35 & 28.85 & 3.95 & 0.97 & 16.49 & 100.71 & 40.28 & 68.23 & 92.35 \\
\hline II & 35.10 & 111.33 & 43.72 & 226.09 & 25.77 & 5.44 & 2.54 & 17.53 & 96.59 & 38.78 & 64.81 & 82.15 \\
\hline III & 33.56 & 110.50 & 44.97 & 269.62 & 27.06 & 4.24 & 1.37 & 17.74 & 91.99 & 36.95 & 67.13 & 87.68 \\
\hline IV & 33.39 & 111.06 & 47.20 & 221.15 & 28.01 & 5.37 & 2.85 & 18.82 & 114.38 & 49.13 & 65.65 & 89.27 \\
\hline V & 35.00 & 111.00 & 47.53 & 230.86 & 19.07 & 4.50 & 1.53 & 22.27 & 91.41 & 34.48 & 63.50 & 80.70 \\
\hline VI & 33.33 & 112.67 & 40.00 & 269.80 & 18.40 & 3.77 & 4.07 & 26.73 & 94.06 & 37.20 & 71.11 & 90.37 \\
\hline VII & 34.33 & 111.00 & 42.80 & 208.35 & 27.00 & 6.57 & 2.20 & 13.27 & 93.76 & 31.20 & 63.42 & 76.31 \\
\hline VIII & 30.33 & 110.33 & 46.33 & 245.72 & 37.40 & 5.33 & 1.10 & 25.67 & 129.40 & 52.77 & 66.36 & 90.19 \\
\hline IX & 33.50 & 112.33 & 43.78 & 210.53 & 18.20 & 6.53 & 4.35 & 24.12 & 88.46 & 35.73 & 68.77 & 83.95 \\
\hline X & 33.33 & 112.33 & 44.87 & 223.91 & 28.73 & 5.73 & 1.20 & 17.73 & 73.62 & 33.58 & 71.86 & 89.96 \\
\hline Mean & 33.27 & 111.20 & 44.45 & 231.63 & 25.84 & 5.14 & 2.21 & 20.03 & 97.34 & 39.01 & 67.08 & 86.29 \\
\hline
\end{tabular}

Table.3 Cont...

\begin{tabular}{|c|c|c|c|c|c|c|c|c|c|}
\hline Cluster No. & DHY & HI & KYP & OIL & PRO & SC & TSS & TFA & PYP \\
\hline I & 25.13 & 43.22 & 11.98 & 47.73 & 25.38 & 3.32 & 12.87 & 0.88 & 17.72 \\
\hline II & 25.83 & 48.30 & 13.81 & 47.28 & 25.16 & 1.77 & 6.37 & 0.91 & 21.37 \\
\hline III & 22.27 & 48.45 & 12.26 & 47.49 & 24.86 & 1.57 & 10.01 & 0.67 & 19.68 \\
\hline IV & 31.67 & 48.06 & 17.48 & 47.43 & 25.80 & 3.20 & 11.99 & 0.76 & 24.26 \\
\hline V & 17.07 & 55.89 & 15.27 & 47.55 & 25.20 & 3.64 & 10.27 & 0.77 & 21.47 \\
\hline VI & 24.00 & 56.22 & 17.73 & 48.00 & 24.85 & 1.37 & 6.86 & 0.82 & 26.33 \\
\hline VII & 26.40 & 42.82 & 11.93 & 47.55 & 25.75 & 3.69 & 11.39 & 0.43 & 18.47 \\
\hline VIII & 28.20 & 51.63 & 17.27 & 47.80 & 26.00 & 2.16 & 14.15 & 0.62 & 24.33 \\
\hline IX & 19.97 & 58.93 & 16.33 & 47.38 & 25.28 & 1.57 & 13.98 & 0.74 & 23.83 \\
\hline X & 31.47 & 42.08 & 10.00 & 47.65 & 25.70 & 3.23 & 6.78 & 0.61 & 20.87 \\
\hline Mean & 25.20 & 49.56 & 14.40 & 47.58 & 25.39 & 2.55 & 10.46 & 0.72 & 21.83 \\
\hline
\end{tabular}

\begin{tabular}{|l|l|l|l|l|l|l|l|l|}
\hline DF & $:$ & Days to 50\% flowering & NMP & $:$ & $\begin{array}{l}\text { Number of mature } \\
\text { pods per plant }\end{array}$ & KYP & $:$ & $\begin{array}{l}\text { Kernel yield per plant } \\
(\mathbf{g})\end{array}$ \\
\hline DM & $:$ & Days to maturity & $100-\mathrm{PW}$ & $:$ & $\begin{array}{l}\text { Hundred pod weight } \\
(\mathrm{g})\end{array}$ & OIL & $:$ & Oil content $(\%)$ \\
\hline SCMR & $:$ & $\begin{array}{l}\text { SPAD chlorophyll meter } \\
\text { reading }\end{array}$ & $100-\mathrm{KW}$ & $:$ & $\begin{array}{l}\text { Hundred kernel } \\
\text { weight }(\mathrm{g})\end{array}$ & PRO & $:$ & Protein content $(\%)$ \\
\hline SLA & $:$ & Specific leaf area $\left(\mathrm{cm}^{2} \mathrm{~g}^{-1}\right)$ & SP & $:$ & Shelling per cent & SC & $:$ & $\begin{array}{l}\text { Sucrose content }(\mathrm{g} \\
\left.100 \mathrm{~g}^{-1}\right)\end{array}$ \\
\hline PH & $:$ & Plant height $(\mathrm{cm})$ & SMK\% & $:$ & $\begin{array}{l}\text { Sound mature kernel } \\
\text { per cent }\end{array}$ & TSS & $:$ & $\begin{array}{l}\text { Total soluble sugars } \\
(\%)\end{array}$ \\
\hline NPB & $:$ & $\begin{array}{l}\text { Number of primary } \\
\text { branches per plant }\end{array}$ & DHY & $:$ & $\begin{array}{l}\text { Dry haulms yield per } \\
\text { plant }(\mathrm{g})\end{array}$ & TFA & $:$ & $\begin{array}{l}\text { Total free amino acids } \\
\left(\mu \mathrm{g}^{-1}\right)\end{array}$ \\
\hline NSB & $:$ & $\begin{array}{l}\text { Number of secondary } \\
\text { branches per plant }\end{array}$ & HI & $:$ & Harvest index $(\%)$ & PYP & $:$ & Pod yield per plant $(\mathrm{g})$ \\
\hline
\end{tabular}


Table.4 Relative contribution of various characters to genetic diversity

\begin{tabular}{|c|c|c|c|}
\hline $\begin{array}{c}\text { S. } \\
\text { No. }\end{array}$ & Character & $\begin{array}{c}\text { No. of times ranked } \\
\text { first }\end{array}$ & $\begin{array}{c}\text { \% } \\
\text { Contribution }\end{array}$ \\
\hline $\mathbf{1}$ & Days to 50 \% flowering & 0 & $0 \%$ \\
\hline $\mathbf{2}$ & Days to maturity & 0 & $0 \%$ \\
\hline $\mathbf{3}$ & SPAD chlorophyll meter reading at 45 DAS & 0 & $0 \%$ \\
\hline $\mathbf{4}$ & Specific leaf area at 45 DAS $\left(\mathrm{cm}^{2} \mathrm{~g}^{-1}\right)$ & 17 & $3.91 \%$ \\
\hline $\mathbf{5}$ & Plant height $(\mathrm{cm})$ & 0 & $0 \%$ \\
\hline $\mathbf{6}$ & Number of primary branches per plant & 1 & $0.23 \%$ \\
\hline $\mathbf{7}$ & Number of secondary branches per plant & 91 & $20.92 \%$ \\
\hline $\mathbf{8}$ & Number of mature pods per plant & 5 & $1.15 \%$ \\
\hline $\mathbf{9}$ & Hundred pod weight $(\mathrm{g})$ & 11 & $2.53 \%$ \\
\hline $\mathbf{1 0}$ & Hundred kernel weight $(\mathrm{g})$ & 3 & $0.69 \%$ \\
\hline $\mathbf{1 1}$ & Shelling per cent & 0 & $0 \%$ \\
\hline $\mathbf{1 2}$ & Sound mature kernel per cent & 81 & $18.62 \%$ \\
\hline $\mathbf{1 3}$ & Dry haulms yield per plant $(\%)$ & 3 & $0.69 \%$ \\
\hline $\mathbf{1 4}$ & Harvest index $(\%)$ & 0 & $0 \%$ \\
\hline $\mathbf{1 5}$ & Kernel yield per plant $(\mathrm{g})$ & 1 & $0.23 \%$ \\
\hline $\mathbf{1 6}$ & Oil content $(\%)$ & 0 & $0 \%$ \\
\hline $\mathbf{1 7}$ & Protein content $(\%)$ & 1 & $0.23 \%$ \\
\hline $\mathbf{1 8}$ & Sucrose content $\left(\mathrm{g} 100 \mathrm{~g}^{-1}\right)$ & 55 & $12.64 \%$ \\
\hline $\mathbf{1 9}$ & Total soluble sugars $(\%)$ & 13 & $2.99 \%$ \\
\hline $\mathbf{2 0}$ & Total free amino acids $\left(\mu \mathrm{g} \mathrm{g} \mathrm{g}^{-1}\right)$ & 149 & $34.25 \%$ \\
\hline $\mathbf{2 1}$ & Pod yield per plant $(\mathrm{g})$ & 4 & $0.92 \%$ \\
\hline
\end{tabular}

\section{Acknowledgment}

Authors are thankful to Acharya N. G. Ranga Agricutural University for providing necessary facilities. Also special thanks to Department of Genetics and Plant Breeding, S. V. Agricultural College, Tirupati.

\section{References}

Choudhary, M A Z., Mia, M F U., Afzal, M A., Ali, M M. 1998. Comparative study of D2 and metroglyph analysis in groundnut. (Arachis hypogaea L.), Thailand J Agric. Sci; 31(3):436-443.

Ganvit, R. S., Jagtap, P. K., Patel, M. C and Malaviya, A. 2018. Genetic divergence studies for yield and its component traits in groundnut (Arachis hypogaea
L.).Journal of Pharmacognosy and Phytochemistry. 7(5): 3056-3058.

Hampannavar, R. M and Khan, H. 2018. Analysis of genetic diversity of groundnut (Arachis hypogaeaL.) genotypes collected from various parts of India. Journal of Pharmacognosy and Phytochemistry. 7(2): 1100-1103.

Mahalanobis, P. C. A statistical study at Chinese head measurement. J Asiatic Soc. Bengal. 1936; 25:301-77.

Namrata., Sharma., Hemlatha., Bisen., Prashant., Singh., Bhumica., Jain and Surbhi. 2018. Assessment of genetic diversity among groundnut (Arachis hypogaea L.) genotypes. International Journal of Bio-Resource and Stress Management. 9(3): 383-386.

Raghuwanshi, S. S., Kachhadia, V. H., 
Vachhani, J. H., Jivani, L. L and Patel, M.B. 2016. Genetic divergence in groundnut (Arachis hypogaea L.). Electronic Journal of Plant Breeding. 7(1): 145-151.

Rao, C. R. 1952. Advanced statistical methods in biometrical research. John Wiley and Sons.New York. 236-272.

Sardar, S. S., Pradhan, K and Behera, B. S. 2017. Divergence study in groundnut breeding lines. Journal of Pharmacognosy and Phytochemistry. 6(5): 1961-1965.

Saritha, K., Vasanthi, R. P., Shanthi Priya, M and Latha, P. 2018. Genetic divergence studies in groundnut (Arachis hypogaea L.). Electronic Journal of Plant Breeding. 9(4): 1355-1361.

Singh, R. K and Chaudhary, B. D. 1977. Biometrical methods in quantitative genetic analysis. Kalyani Publishers, New Delhi. 215-218.

Sudhakar, B. V. 2008. Genetic divergence for yield, physiological and quality traits in groundnut (Arachis hypogaea L.). M.Sc. Thesis. Acharya N G Ranga
Agricultural University, Hyderabad, India.

Vasanthi, R. P., Suneetha, N and Sudhakar, P. 2015b. Genetic diversity based on physiological attributes among released and pre-release cultures of groundnut (Arachis hypogaea L.). Legume Research. 38(1): 47-50.

Venkatesh, K., Rajesh, A. P., Srinivas, T and Umamaheswari. P. 2016.Assessment of genetic diversity for kernel yield and quantitative traits in drought tolerant groundnut genotypes. Electronic Journal of Plant Breeding. 7(1): 29-36.

Venkateswarulu, O., Sudhakar, B. V. G., Reddi, M. S and Sudhakar, P. 2011. Genetic divergence in confectionary types of groundnut (Arachis hypogaea L.). Legume Research. 34(1): 1-7.

Zaman, M. A., Thuhina, M. K., Bhuiyan, M. M. H., Moniruzzamn, $\mathrm{M}$ and Yousuf, M. N. 2010. Genetic divergence in groundnut (Arachis hypogaea L.). Bangladesh Journal of Plant Breeding and Genetics. 23(1): 45-49.

\section{How to cite this article:}

Aruna Kumari, E., K. John, D. Mohan Reddy and Latha, P. 2021. Genetic Divergence Analysis in Advanced Breeding Lines of Groundnut (Arachis hypogaea L.). Int.J.Curr.Microbiol.App.Sci. 10(05): 236-242. doi: https://doi.org/10.20546/ijcmas.2021.1005.031 